\title{
Initial Measurement of Doppler-Shifted DD Neutron Energy Spectrum Using CLYC7 Scintillator in LHD
}

\author{
Yasuko KAWAMOTO $^{1)}$, Kunihiro OGAWA ${ }^{1,2)}$, Mitsutaka ISOBE ${ }^{1,2)}$, Siriyaporn SANGAROON ${ }^{3)}$, \\ Guoqiang $\mathrm{ZHONG}^{4)}$ and Masaki OSAKABE ${ }^{1,2)}$ \\ 1) National Institute for Fusion Science, National Institutes of Natural Sciences, 322-6 Oroshi-cho, Toki, Gifu 509-5292, \\ Japan \\ 2) The Graduate University for Advanced Studies, SOKENDAI, 322-6 Oroshi-cho, Toki, Gifu 509-5292, Japan \\ ${ }^{3)}$ Faculty of Science, Mahasarakham University, Maha Sarakham, 44150, Thailand \\ 4) Institute of Plasma Physics, Chinese Academy of Sciences, Hefei, 230031, People's Republic of China
}

(Received 1 November 2021 / Accepted 16 November 2021)

\begin{abstract}
The compact neutron energy spectrometer (CNES), based on the $\mathrm{Cs}_{2} \mathrm{LiYCl}_{6}$ : Ce with a ${ }^{7} \mathrm{Li}$-enrichment (CLYC7) scintillator, has been developed for a neutron energy diagnostic in large helical device (LHD) plasma in the 2020 experimental campaign. The CNES, installed on a tangential sightline, is utilized to measure neutron energy spectra from tangentially-injected negative-ion-based neutral beam (N-NB) heated deuterium plasmas. In this paper, an initial result of the Doppler-shifted neutron spectrum is reported.
\end{abstract}

(c) 2021 The Japan Society of Plasma Science and Nuclear Fusion Research

Keywords: neutron energy spectrometer, LHD, Doppler shift of neutron energy, deuterium plasma, CLYC7

DOI: $10.1585 /$ pfr. 16.1202108

Energetic ions have important roles in fusion plasma experiments in terms of plasma heating, current drive etc. Thus the understanding of energetic ion behavior is necessary. In the large helical device (LHD), a fast ion Dalpha diagnostic [1], a tangential E\|B-type neutral particle analyzer [2], and a scintillator-based lost-fast ion probe [3] are employed as an energetic ion diagnostic. Neutron diagnostics provide information on energetic ions in deuteron plasmas because neutrons are mainly produced by so-called beam-thermal reactions in LHD deuterium plasmas [4]. The total neutron emission rate, which reflects a global confinement of beam ions, has been measured using the neutron flux monitor (NFM) [5]. Also, the neutron emission profile, which provides a spatial profile of beam ions, has been measured using vertical neutron cameras [6]. Recently, to obtain deep understanding of energetic ion behavior, a compact neutron energy spectrometer (CNES) has been newly installed with a tangential sightline.

The CNES is based on the $\mathrm{Cs}_{2} \mathrm{LiYCl}_{6}$ : Ce with a ${ }^{7} \mathrm{Li}-$ enrichment (CLYC7) scintillator (1-inch $\phi$ x 1-inch height) coupled with a photo-multiplier tube. The CLYC7 scintillator utilizes the ${ }^{35} \mathrm{Cl}(\mathrm{n}, \mathrm{p}){ }^{35} \mathrm{~S}$ reactions to measure energy of the Deuterium-Deuterium (DD) neutrons. Note that the cross section of this reaction monotonically increases from $100 \mathrm{keV}$ up to $10 \mathrm{MeV}$ and we set the discrimination level of data acquisition system at $500 \mathrm{keV}$ in the neutron energy to cut off the influence of resonance in the cross section. Besides an enrichment in ${ }^{7} \mathrm{Li}$ suppresses the sensitiv-

author'se-mail: kawamoto.yasuko@nifs.ac.jp ity to thermal-neutrons because the ${ }^{6} \mathrm{Li}(\mathrm{n}, \alpha) \mathrm{T}$ reaction has a large cross section for thermal neutron. Thus this reaction is suitable for DD neutron measurement. Energy calibration of the CNES was performed by use of an acceleratorbased neutron source at the Fast Neutron Laboratory of Tohoku university [7]. As shown in Fig. 1, the CNES was installed with a tangency radius $\sim 3.65 \mathrm{~m}$ on the midplane and was immersed in a neutron collimator composed of borated polyethylene for neutron shielding, and lead for gamma-ray shielding. The signal of the CNES was directory fed into a fast digitizer (DT5720B, CAEN).

Figure 2 shows the time evolution of a deuterium

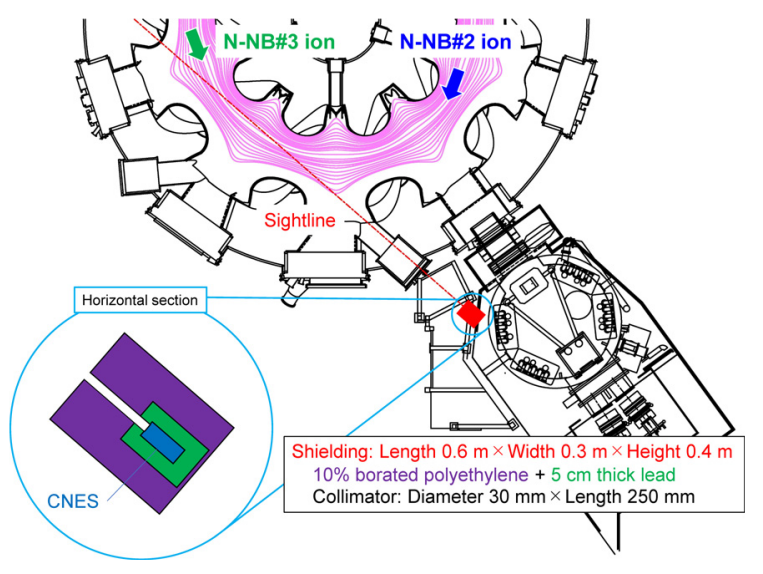

Fig. 1 Schematic top view of LHD and CNES, together with sightline of CNES and direction of N-NBs. 


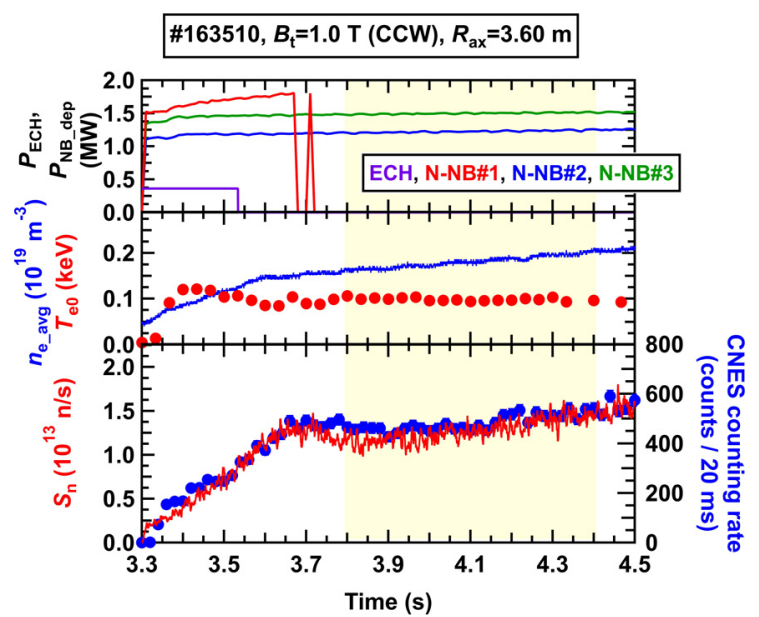

Fig. 2 Time evolution of injection power of Electron Cyclotron Heating $(\mathrm{ECH})$, deposition power of N-NBs, lineaveraged electron density $n_{e_{-} a v g}$, central electron temperature $T_{e 0}$, total neutron emission rate measured by NFM, and pulse counting rate measured by CNES for \#163510.

beam-heated deuterium plasma discharge. This discharge was operated under the magnetic configuration of $R_{a x}=$ $3.60 \mathrm{~m}$. The magnetic field strength $B_{t}=1.0 \mathrm{~T}$ in a counterclockwise $(\mathrm{CCW})$ direction is seen from the top of the LHD. The injection energy of both N-NB\#2 and N-NB\#3 is $160 \mathrm{keV}$. As shown in the bottom of Fig. 2, the time evolution of the pulse counting rate obtained by the CNES followed a total neutron emission rate $\left(S_{n}\right)$ measured by the NFM, indicating the CNES is away from pile-up of pulse signals. We selected a stable plasma phase $t=3.8$ - $4.4 \mathrm{~s}$, where $n_{e \_a v g}$ and $T_{e 0}$ were almost unchanged, for neutron energy spectra measurement. In this stable plasma phase, N-NB\#2 was injected clockwise-direction and $\mathrm{N}$ NB\#3 was injected CCW-direction, simultaneously. The $\mathrm{N}-\mathrm{NB} \# 2$ ions moved away from the CNES, whereas $\mathrm{N}$ NB\#3 ions moved toward the CNES, as shown in Fig. 1. The neutron energy spectrum obtained by the CNES is shown in Fig. 3. Here the error bar shows a statistical error in counts. Fitting with two gaussian functions shows that energy peaks appeared around $2.3 \mathrm{MeV}$ and $2.9 \mathrm{MeV}$ in the spectrum. The lower peak corresponds to N-NB\#2 ions and higher peak does to N-NB\#3 ions because those are red-shifted and blue shifted from the DD neutron energy (2.45 MeV) in the center of mass system, respectively.

To identify the two peaks appearing in Fig. 3, they are compared with energy estimated using a two-body kinematic calculation. The kinetic energy of the DD neutron $\left(E_{n}\right)$ in the laboratory system [8] is described,

$$
\begin{aligned}
E_{n} & =\frac{1}{2} m_{n} V_{c}^{2}+\frac{m_{3 H e}}{m_{3 H e}+m_{n}}\left(Q_{D D}+E_{r}\right) \\
& +V_{c} \cos \theta_{c} \sqrt{\frac{2 m_{3 H e} m_{n}}{m_{3 H e}+m_{n}}\left(Q_{D D}+E_{r}\right)},
\end{aligned}
$$

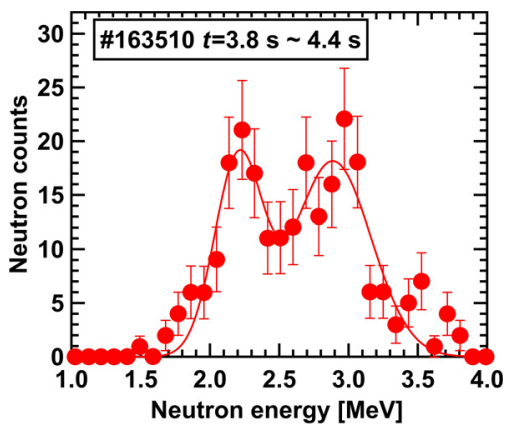

Fig. 3 Neutron spectra accumulated in $t=3.8 \sim 4.4 \mathrm{~s}$ obtained by CNES. Closed circles represent experimental data and solid line represents gaussian fitting of experimental data.

where $m_{3 \mathrm{He}}$ and $m_{n}$ are the mass of ${ }^{3} \mathrm{He}$ and the neutron, respectively, $V_{C}$ is the center-of-mass velocity of reactant deuterons, $E_{r}$ is the relative energy between the deuterons, $Q_{D D}$ is the reaction $Q$-value for the $\mathrm{D}(\mathrm{d}, \mathrm{n})^{3} \mathrm{He}$ reaction (i.e., $3.27 \mathrm{MeV}$ ), and $\theta_{C}$ is the angle between the direction of center-of-mass and the direction of the emitted neutron. In this case, $\theta_{C}=140$ degrees and 45 degrees for N-NB\#2 and N-NB\#3, respectively because the tangency radius of $\mathrm{N}-\mathrm{NB} \# 2$ is $3.75 \mathrm{~m}$ and the tangency radius of $\mathrm{N}-\mathrm{NB} \# 3$ is $3.70 \mathrm{~m}$. The value of $E_{r}$ is set to be the injection energy of N-NBs, e.g., $160 \mathrm{keV}$. From Eq. (1), $E_{n}$ for N-NB\#2 ion is $2.2 \mathrm{MeV}$, whereas N-NB\#3 ion is $2.9 \mathrm{MeV}$. The measured Doppler shift of DD neutron energies is almost consistent with the analysis based on two-body DD neutron energy calculation using the tangency radius of the CNES sight line, injection energies and tangency radii of N-NBs. Further analyses considering the energy slowingdown process of energetic particles produced by N-NBs and the effect of their drift orbits are necessary for accurate evaluation of the neutron energy spectrum.

In summary, for the understanding of energetic ion behavior, we newly installed a tangential CNES, based on the CLYC7 scintillator in the LHD. The Doppler-shifted DD neutron spectrum was successfully measured by the CNES in a N-NB heated deuterium plasma discharge. The measured peak positions in the neutron spectrum are consistent with analytically evaluated Doppler-shifted neutron energy corresponding to N-NB\#2 and N-NB\#3.

[1] Y. Fujiwara et al., Nucl. Fusion 80, 112014 (2020).

[2] S. Kamio et al., Nucl. Fusion 60, 112002 (2020).

[3] K. Ogawa et al., Nucl. Fusion 50, 084005 (2010).

[4] M. Osakabe et al., Fusion Sci. Technol. 72, 199 (2017).

[5] M. Isobe et al., Trans. Plasma Sci. 46, 2050 (2017).

[6] K. Ogawa et al., Nucl. Fusion 61, 096035 (2021).

[7] M. Baba et al., Nucl. Instrum. Methods Phys. A 376, 115 (1996).

[8] J. Scheffel, Nucl. Instrum. Methods Phys. Res. 224, 519 (1984). 